\title{
The Importance of Treating Functional Cross Bite: A Clinical Viewpoint
}

\author{
Prabhakar $\mathbf{M}^{1 *}$, Nindra $\mathrm{J}^{2}$, Sehrawat $\mathrm{S}^{3}$ and Singh $\mathrm{S}^{3}$ \\ ${ }^{1}$ Professor, Faculty of Dental Sciences, SGT University, India \\ ${ }^{2}$ Senior Lecturer, Faculty of Dental Sciences, SGT University, India \\ ${ }^{3}$ Postgraduate student, Department of Orthodontics \& Dentofacial Orthopedics, SGT University, \\ India
}

\section{Case Report}

Volume 6 Issue 3

Received Date: July 01, 2021

Published Date: July 23, 2021

DOI: $10.23880 /$ oajds-16000301

*Corresponding author: Mona Prabhakar, Professor, Faculty of Dental Sciences, SGT University, Gurgaon, Haryana, 122505, India, Tel: 9810131064; Email: mona.prabhakar@sgtuniversity.org

\section{Abstract}

Functional cross bites can bring about structural changes on account of asymmetric condylar positioning and neuromuscular patterns. In the long term uneven bite forces and asymmetric EMG loading patterns can lead to mandibular asymmetry, periodontal damage and poor esthetics. Moving the involved teeth into optimal functional positions can normalize the path of mandibular closure and eliminate asymmetric neuromuscular function. It is important that these changes are done early, during the mixed dentition stage, to minimize factors that are detrimental to harmonious occlusion and jaw growth. However, sometimes due to insufficient awareness, the visit to the dental office for regular checkups does not take place until signs of facial asymmetry or gross irregularity in the alignment of anterior teeth are evident. The adolescent is well into the permanent dentition by then and has a firmly established forward path of closure of the mandible which is not easy to alter. Eliminating the forward slide of mandible even at this stage can position the condyles symmetrically within the glenoid fossae. Establishing a positive overjet can help to limit the unrestricted mandibular growth that might have occurred, had the reverse incisor relationship persisted.

Keywords: Functional cross bite; Mandibular asymmetry; Adverse effects

Abbreviations: AAO: American Association of Orthodontists.

\section{Introduction}

A cross bite is defined by American Association of Orthodontists (AAO) as a type of malocclusion or a misalignment of teeth, where upper teeth fit inside the lower teeth when the jaws are brought together into occlusion. This misalignment can affect a single tooth or groups of teeth and can occur in the anterior, posterior or both regions of the dental arch.
It is a commonly occurring condition in the mixed dentition which is also the age for rapid growth of the alveolar processes, maxillary and mandibular jaw bones and the attached muscles. Cross bites can bring about structural or anatomical changes on account of asymmetric condylar positioning and neuromuscular patterns. In the long term uneven bite forces, asymmetric EMG loading patterns on both sides of the mandible can lead to asymmetries in mandibular length [1]. In cases of anterior cross bite, esthetics are poor and if the condition is not treated it can lead to tissue damage in the form of attrition of teeth in cross bite, gingival recession, loss of alveolar bone support 


\section{Open Access Journal of Dental Sciences}

and mobility of the lower incisor tooth [2]. For the reasons cited above the correction of cross bites by moving the involved teeth into optimal functional positions can restore normal closure pattern of the mandible thereby eliminating asymmetric condylar positions and neuromuscular function. It is important that these changes are done immediately on detection of the problem to minimize factors that are detrimental to harmonious occlusion and jaw growth.

In a prospective study done by Pinto \& Buschang, et al. on the morphological and positional asymmetries of young children with functional unilateral posterior cross bite, the results showed that the mandible was significantly longer on the non-cross bite side with the asymmetry being most pronounced in the ramal region besides involving the condylar and the coronoid processes. However after treatment and retention, the morphological asymmetries disappeared with greater growth occurring on the cross bite than on the noncross bite side and the mandible apparently being realigned [3]. They also evaluated the changes in masticatory cycle shape and duration following rapid palatal expansion and found that treatment shortened the cycle duration but did not alter the abnormal chewing cycle shape [4].

Peter LH, et al. did a retrospective study to determine mandibular asymmetries using submentovertex radiographs. They also tried to evaluate whether condylar position in children with functional unilateral cross bite was different from that found in children with Class I noncross bite malocclusions and if there was a change in condylar position following correction of cross bite by palatal expansion. Mandibles of children in the functional cross bite group exhibited asymmetry in both anteroposterior and transverse dimensions when compared with Class I non-cross bite group along with a Class II subdivision occlusal relationship on the cross bite side. Examination of the condylar positions before and after treatment demonstrated large standard deviations and hence provided no conclusive evidence of any significant difference in condylar positions between or within the groups before and after treatment [5]. Myers, et al. tried to determine whether functional posterior cross bites in children influence the position of the mandibular condyle and assessed the effect of cross bite correction on condylar position using transcranial temporomandibular joint radiographs both prior to treatment with the teeth occluded in cross bite position and following cross bite correction with the teeth in centric occlusion. They found significant differences in the horizontal and vertical joint space measurements between the cross bite and noncross bite sides while the significant differences disappeared on cross bite correction [6].

Korbmacher, et al. have even suggested associations between orthopedic disturbances and unilateral cross bite in children with asymmetry of the upper cervical spine although there was no evidence of a causal link [7].

\section{Establishing a Differential Diagnosis}

A cross bite maybe skeletal, dentoalveolar or functional in nature, making it important to diagnose the underlying structural deviation before a treatment plan is formulated. Sometimes a cephalometric assessment may not be the most reliable tool to differentiate whether the maxilla or the mandible contribute to the skeletal disharmony. Instead the dental characteristics seem to be more consistent findings which include Class III molar and canine relationships lingually inclined mandibular incisors, proclined maxillary incisors and an edge to edge incisor relationship or anterior cross bite. In case of anterior cross bite, to differentiate a dental from a skeletal problem, Ngan, et al. have proposed a diagnostic scheme consisting of a dental assessment followed by a functional assessment and finally a profile analysis [8].

While studying the diagnostic criteria of pseudo Class III malocclusion in a southern Chinese population, Rabie concluded that compared with Class I malocclusion a pseudoClass III malocclusion is characterized by retroclined upper incisors but normally inclined lower incisors, retrusive upper lip, decreased midface length, forward position of the mandible with normal mandibular length and increased maxillary-mandibular difference. Majority $(72 \%)$ of the cases with pseudo- Class III malocclusion had no family history and showed a Class I molar relationship at habitual occlusion [9].

Likewise it is important to differentiate whether a unilateral posterior cross bite is just dental, skeletal or dentoalveolar associated with a functional lateral shift of the mandible. If only one or two teeth are in cross bite with coinciding mandibular-maxillary dental midlines, the condition can be easily corrected by just moving the teeth in cross bite. However when an entire buccal segment is involved, with non-coincident dental midlines when upper and lower teeth occlude, it is usually due to a bilaterally constricted maxillary arch. The mandible swings laterally to achieve an occlusion of convenience or complete intercuspation on at least one side while the other remains in cross bite. A bilateral posterior cross bite on account of transverse discrepancy of maxillary- mandibular widths is of skeletal nature; is evident both in centric relation and in maximum intercuspation, is of greater severity, exhibits a reduced palatal width to depth ratio as given by Korkhaus and is very difficult to correct.

\section{The Need for Intervention}

The rate of self-correction of cross bites is too low to 
justify non-intervention [10,11]. Posterior cross bites in the deciduous dentition showed self-correction of between $0 \%$ and $95 \%[11,12]$. Removal of functional interferences has been found to be successful only in children under the age of 5 years with success rates ranging from $27 \%$ to $64 \%$ $[10,13,14]$. There is sufficient evidence that untreated crossbites will lead to permanent growth alteration, making early treatment crucial. Kennedy and Osepchook [15] have inferred that the glenoid fossa and condyle will undergo remodeling during growth to compensate for condylar asymmetry if left untreated, although there is no longitudinal research to provide conclusive evidence of glenoid fossa and condylar skeletal adaptation. Pirttiniemi, et al. stressed the need for early correction of lateral malocclusions due to the asymmetric position of the condyles in a CT evaluation of bicondylar position in a group of adult patients, indicating that complete adaptation seldom occurs [16]. However O'Byrn, et al. have cautioned and raised the question whether an orthodontist should correct a unilateral cross bite by orthodontic means alone when it is present in adult patients. Their study using submentovertex radiographs indicated that adaptations both in the position of the mandible and the dentition exist in adults with untreated unilateral posterior cross bites. They surmise that with correction of the occlusal problem there is a possibility of changing the condylar position within the fossa. The sudden change in condylar position may not be matched by the individual's adaptive capacities, leading to discomfort and pain. Hence they recommend longitudinal examination involving pre and post treatment tomograms of children as well as adults who have undergone cross bite corrections to document the condylar position change within the fossa as a result of treatment and to follow them up post treatment to determine if condylar adaptation occurs [17].

Below are presented cross bite correction in two males, aged 19yrs (case1; Figures 1-6) and 16yrs (case 2; Figures 7-9) respectively, both having a large segment of anterior teeth in cross bite. As they reported for orthodontic treatment rather late, well into the permanent dentition stage, they had already developed a certain degree of mandibular asymmetry. In both cases there seemed to be a high chance that what began as a functional class III malocclusion progressed over the years into a skeletal discrepancy on account of not having received timely orthodontic intervention.

In both cases the upper lower arches were dis-occluded using an acrylic posterior bite plate to alleviate the anterior locking of the maxillary teeth within the mandibular arch. Light round multilooped .014 stainless steel arch wires were then used to expand and level the maxillary arch and procline the maxillary teeth to jump the cross bite. This was followed by incorporating push coil springs to create space for the blocked out canine in Case 1. Once the bite was corrected the arches were progressively leveled till .017x025 SS wires in the 18 slot pre adjusted fixed appliance giving a short phase of 5/16" green elastics for 2 months. Occlusion was settled on light.014 NiTi wires for a month and the cases were debonded. Case 1 was recalled 2 years post debonding (Figures 4-6); while Case 2 was recalled 1year post debonding (Figures 10-12) to check the stability of the treatment as well as for a fresh set of photographs and radiographs. The occlusal outcome in each case appeared stable and periodontal health was much improved as compared to its pre-treatment status.

\section{Case 1}

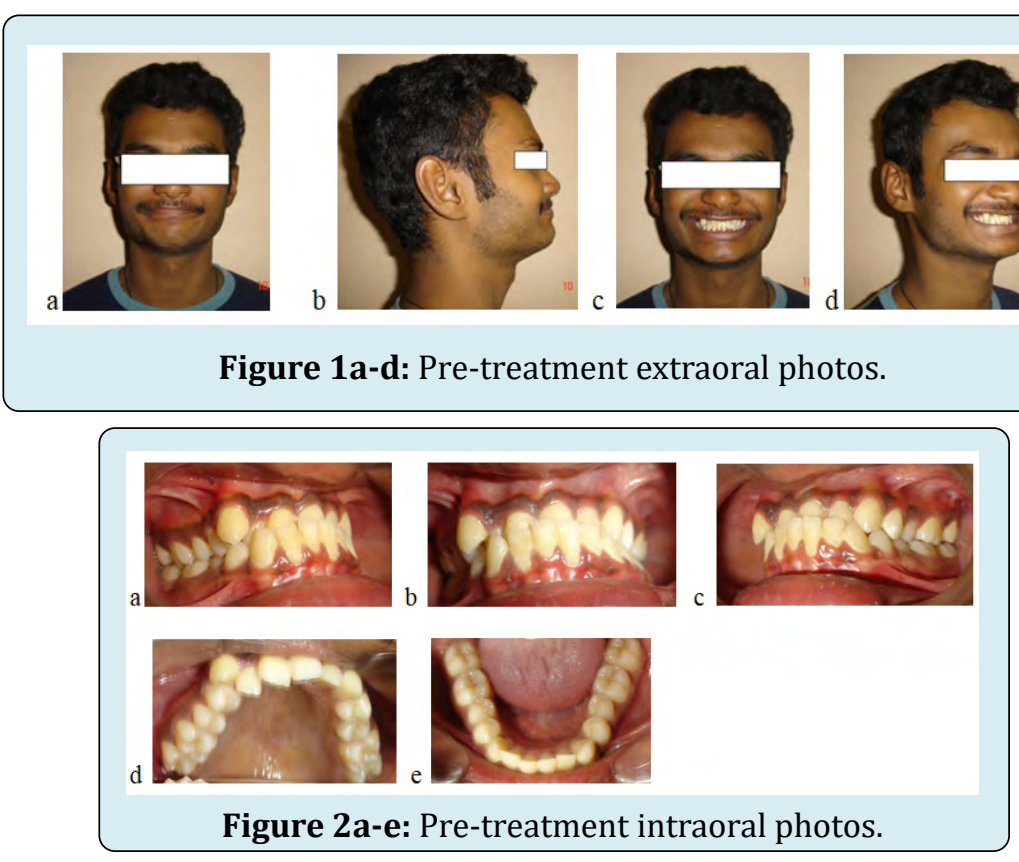



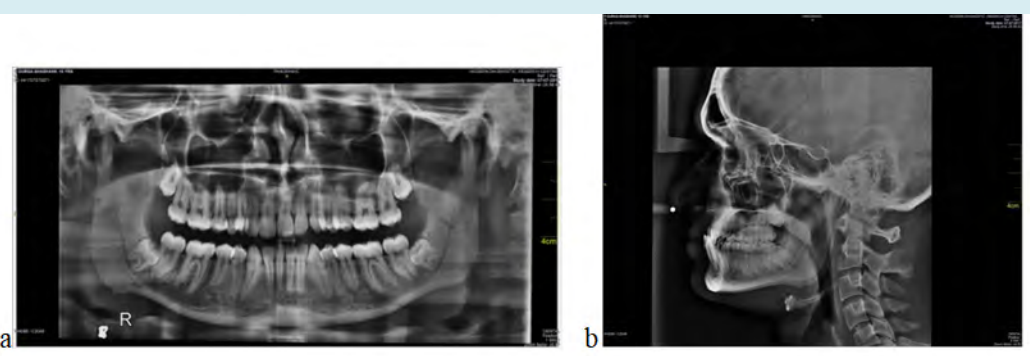

Figure 3a-b: Pre-treatment radiographs.

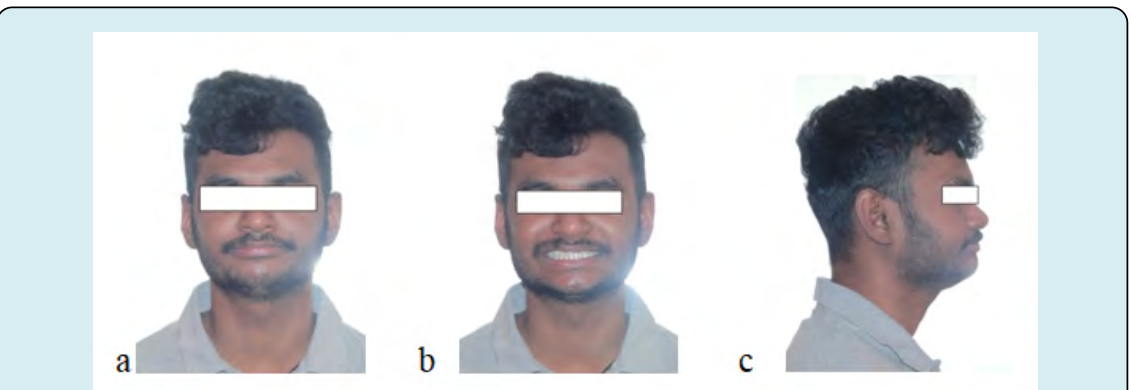

Figure 4a-c: Post-treatment extraoral photos.
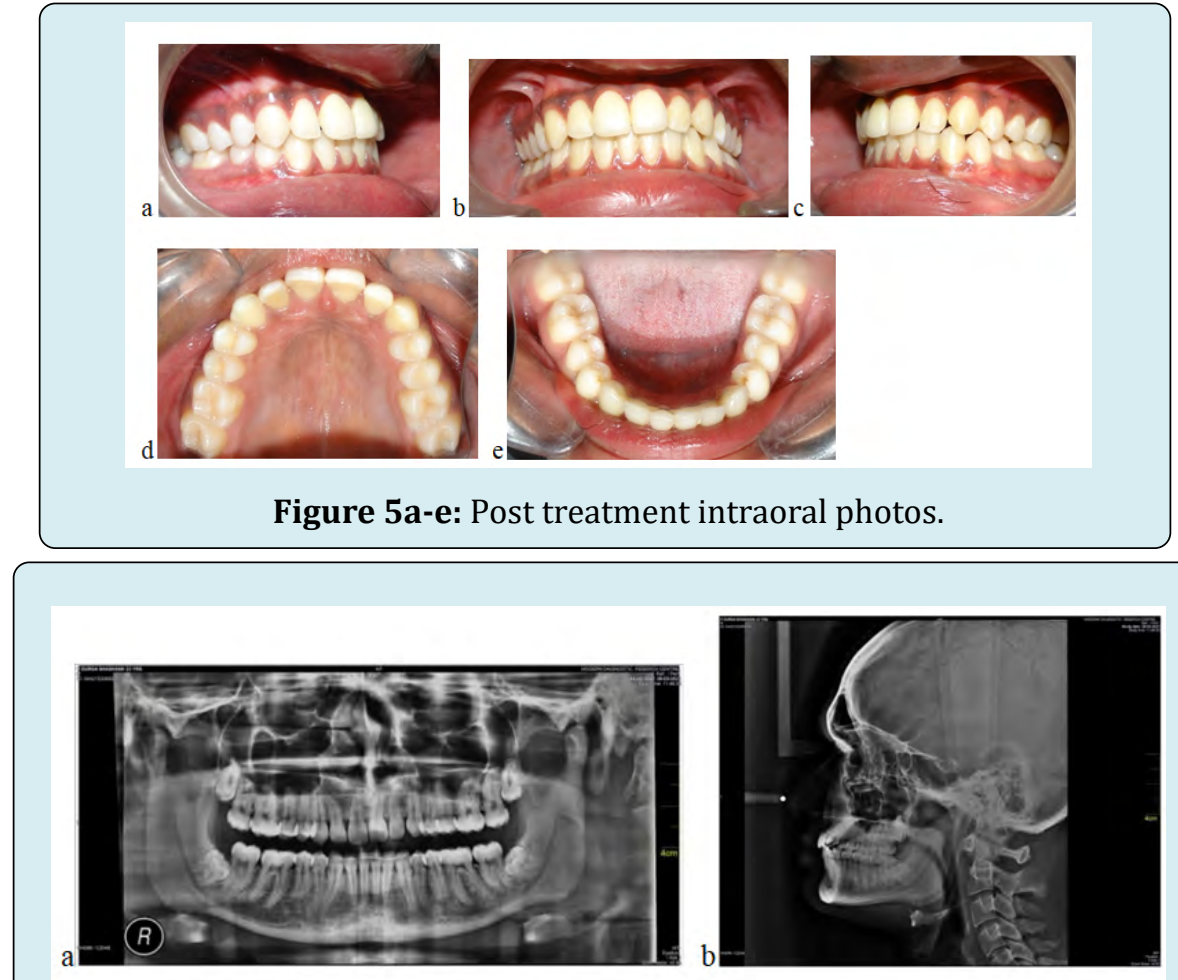

Figure 6a-b: Post-treatment radiographs. 


\section{Case 2}

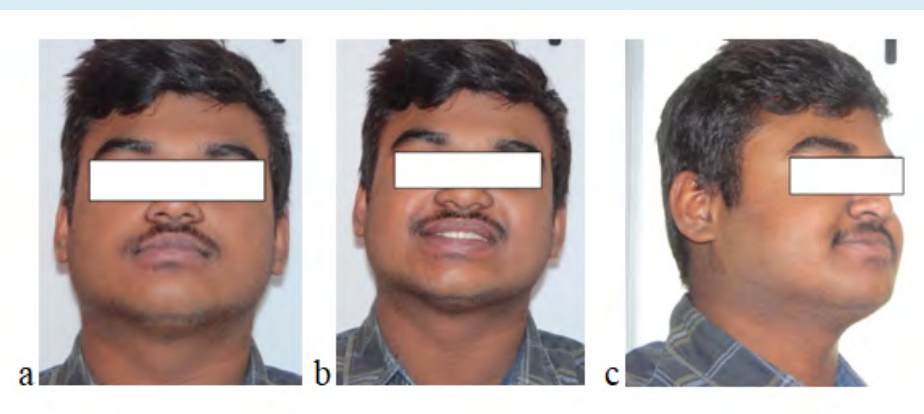

Figure 7a-c: Pre-treatment extraoral photos.
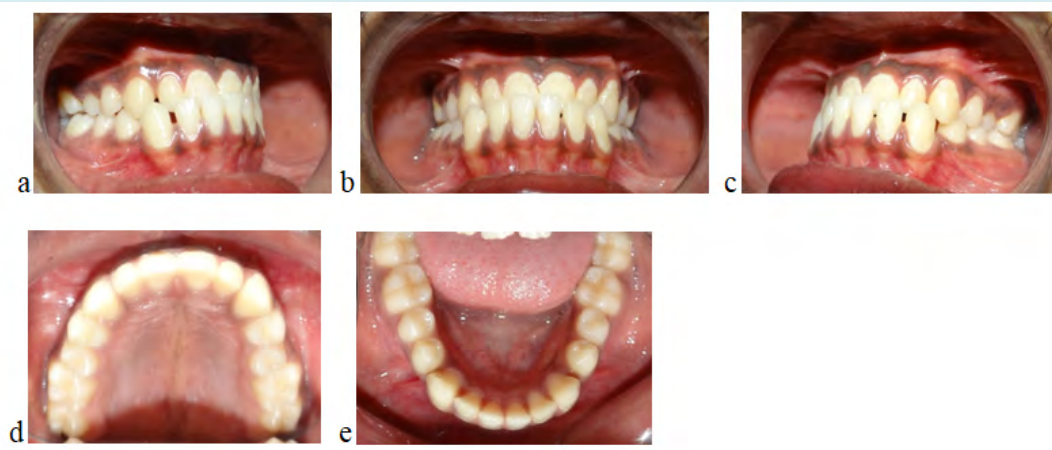

Figure 8a-e: Pre-treatment intraoral photos.
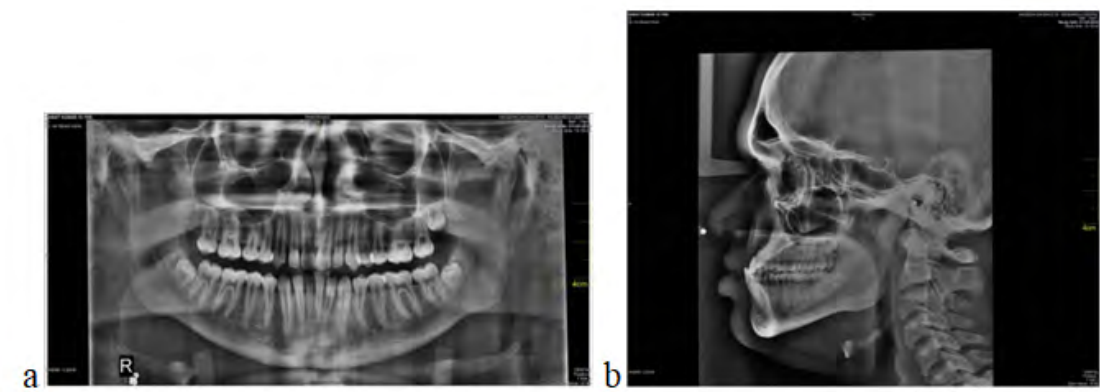

Figure 9a-b: Pre-treatment radiographs.

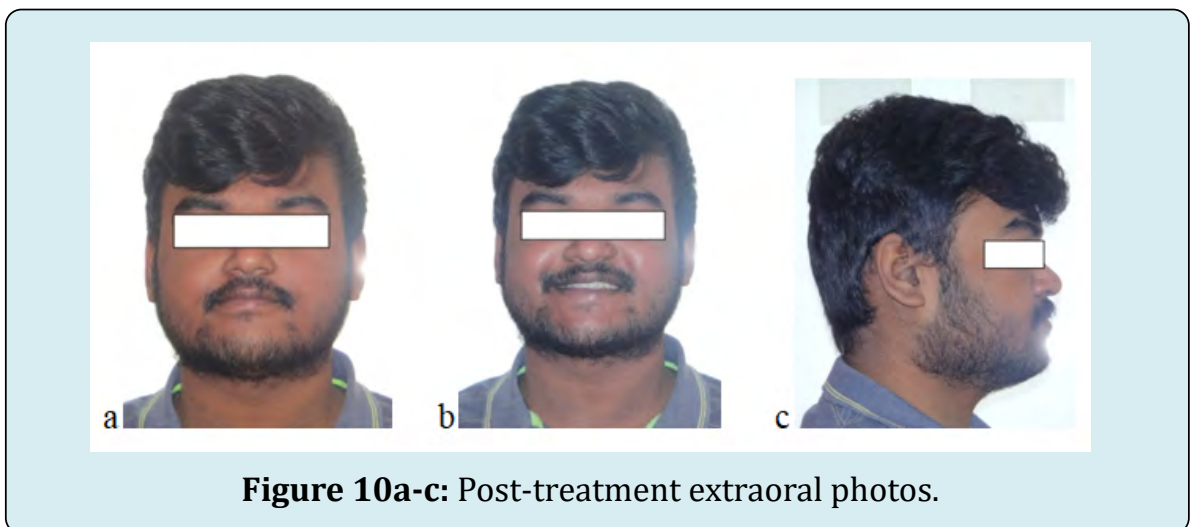



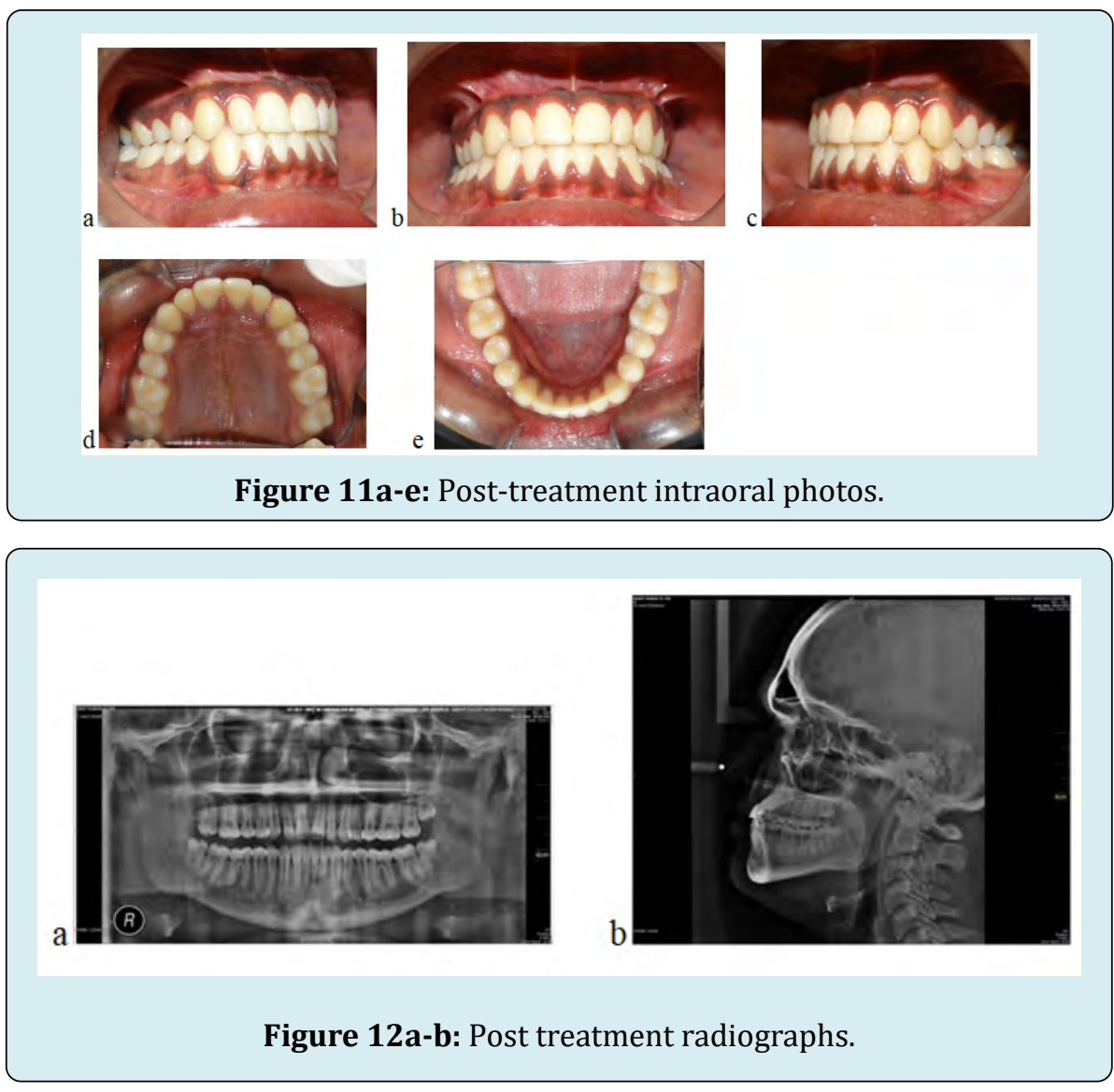

\section{Discussion}

It is best that pseudo Class III occlusion be corrected in the mixed dentition, as early as it is detected or diagnosed. This helps to establish the right incisal guidance for optimal mandibular movements and also removes the inhibition of maxillary growth. However in many instances, owing to insufficient awareness amongst parents, especially in a developing country like India, the visit to the dental office for regular checkups does not take place until there are beginning signs of facial asymmetry, an improper facial form or gross irregularity in the alignment of anterior teeth. The adolescent is by then well into the permanent dentition and has a firmly established forward path of closure of the mandible which is not easy to alter. Owing to years of mastication and deglutition in a reverse bite, the mandibular alveolar process shows alteration in form, akin to skeletal class III malocclusion i.e., increased SNB angle, decreased SNA angle and a negative ANB angle. The deformity may even involve the jaw bases, converting what actually was a pseudo class III occlusion with skeletal class I jaw bases in childhood into a frank skeletal Class III in the adult teenager.

If the jaw base disproportion is mild to moderate it can be camouflaged by tipping the maxillary incisors labially to correct the bite. Many a time establishing a positive overjet, in such cases where there is scope remaining for mandibuar growth, acts as a magical boon. It can help to limit the otherwise unrestricted mandibular growth that would have occurred, had the reverse incisor relationship persisted. It has a remarkably beneficial effect on the periodontal support of the involved teeth and stops the attrition of anterior teeth enamel. Although not proven so far, eliminating forward slide of mandible may restore optimally centred position of the condyles within the glenoid fossae. A comparison of the pre and post treatment OPGs of both cases shown here are suggestive of this finding although it would definitely have been better to confirm the same using tomograms or CBCT of the TMJ before and after treatment.

In the above two cases shown, the profile and cephalogram indicate a strong horizontal growth pattern, decreased lower facial height with forward rotation and over closure of the mandible. The combination of all these features most likely led to a functional Class III closure pattern when the lower incisors erupted and it persisted well into adolescence bringing about some degree of skeletal Class III like changes in the maxilla and mandible. 


\section{Conclusion}

In view of the manifold benefits, every effort should made to correct cross bites that are dentoalveolar, functional or associated with mild skeletal Class III that is suspected to have developed due to an alteration of mandibular function over several years. This condition should be differentiated from mandibular prognathism that has a strong familial tendency, signs of which can be seen at an early age and which shows typical structural features like large gonial angle, steep mandibular plane and large maxillo-mandibular discrepancy.

\section{References}

1. Bell RA, Kiebach T (2014) Posterior cross bites in children: Developmental-based diagnosis and implications to normative growth patterns. Semin Orthod 20(2): 77-113.

2. Borrie F, Bearn D (2011) Early correction of anterior cross bites: a systematic review. J Orthod 38(3): 175184.

3. Pinto AS, Buschang PH, Gaylord S, Throckmorton GS, Chen P (2001) Morphological and positional asymmetries of young children with functional unilateral posterior cross bite. Am J Orthod Dentofac Orthop 120(5): 513-520.

4. Throckmorton GS, Buschang PH, Hayasaki H, Pinto AS (2001) Changes in the masticatory cycle following treatment of posterior unilateral cross bite in children. Am J Orthod and Dentofac Orthop 120(5): 521-529.

5. Lam PH, Sadowsky C, Omerza F (1999) Mandibular asymmetry and condylar position in children with unilateral posterior cross bite. Am J Orthod and Dentofac Orthop 115(5): 569-575.

6. Myers DR, Barenie JT, Bell RA, Williamson EH (1980) Condylar position in children with functional posterior cross bite: before and after cross bite correction. Pediatr Dent 2(3): 190-194.

7. Kormacher H, Koch L, Stroeder ES, Nieke BK (2007)
Associations between orthopedic disturbances and unilateral cross bite in children with asymmetry of the upper cervical spine. Eur J Orthod 29(1): 100-104

8. Ngan P, Hu AM, Fields HW (1997) Treatment of Class III problems begins with differential diagnosis of anterior crossbites Pediatric Dentistry 19(6): 386-395.

9. Rabie ABM, Gu Y (2000) Diagnostic criteria for pseudo Class III malocclusion. Am J Orthod Dentofac Orthop 117(1): 1-9.

10. Kutin G, Hawes RR (1969) Posterior cross-bites in the deciduous and mixed dentitions. Am J Orthod 56(5): 491-504.

11. Schroder U, Schroder I (1984) Early treatment of unilateral posterior cross bite in children with bilaterally contracted maxillae. Eur J Orthod 6(1): 65-69.

12. Thilander B, Wahlund S, Lennartsson B (1984) The effect of early interceptive treatment in children with posterior cross bite. Eur J Orthod 6(1): 25-34.

13. Kurol J, Bergland L (1992) Longitudinal study and cost-benefit analysis of the effect of early treatment of posterior cross bites in the primary dentition. Eur J Orthod 14(3): 173-179.

14. Lindner A (1989) Longitudinal study of the effect of early interceptive treatment in 4 year children with unilateral cross-bite. Scand J Dent Res 97(5): 432-438.

15. Kennedy DB, Osepchook M (2005) Unilateral posterior cross bite with mandibular shift: A Review. J Can Assoc 71(8): 569-573.

16. Pirttiniemi P, Raustia A, Kantomaa T, Pyhtinen J (1991) Relationships of bicondylar position to occlusal symmetry. Eur J Orthod 13(6): 441-445.

17. Obyrn BL, Sadowsky C, Schneider B, BeGole EA (1995) An evaluation of mandibular symmetry in adults with unilateral posterior cross bite. Am J Orthod Dentofacial Orthop 107(4): 394-400. 